\title{
Motor-equivalent covariation stabilizes step parameters and center of mass position during treadmill walking
}

\author{
Julius Verrel • Martin Lövdén · \\ Ulman Lindenberger
}

Received: 15 March 2010/Accepted: 9 September 2010/Published online: 23 September 2010

(C) Springer-Verlag 2010

\begin{abstract}
We investigated motor-equivalent stabilization of task-related variables (TRV) at times of heel strike in eight healthy young men (23-30 years) who walked on a motorized treadmill at self-selected and prescribed speeds within the normal walking speed range. The TRV consisted of step parameters (step length and width) and the center of mass (CoM) position relative to the support (back and front feet). Motor-equivalent stabilization of the TRV was assessed using a decorrelation technique, comparing empirical to decorrelated (covariation-free) variability. Analysis indicated reliable covariation for all TRV. In both the fore-aft and lateral directions, stabilization by covariation was highest for $\mathrm{CoM}$ position relative to the front foot, indicating a prioritization of equilibrium-related variables. Correlations among TRV revealed that the relation between CoM and step parameter control differed between the fore-aft and lateral directions. While stabilization of lateral foot position appears to be due to control of CoM relative to each foot, step length showed small, but reliable, stabilization beyond CoM stabilization, which may be related to spatiotemporal regularity of the step pattern.
\end{abstract}

Keywords Gait variability - Covariation by randomization · Equilibrium · Movement coordination

J. Verrel $(\bowtie) \cdot$ M. Lövdén · U. Lindenberger

Max Planck Institute for Human Development,

Center for Lifespan Psychology, Lentzeallee 94,

14195 Berlin, Germany

e-mail: verrel@mpib-berlin.mpg.de

M. Lövdén

Department of Psychology, Lund University,

Box 213, 22100 Lund, Sweden

$\begin{array}{ll}\text { Abbreviations } & \\ \text { DOF } & \text { Degree(s) of freedom } \\ \text { TRV } & \text { Task-related variable(s) } \\ \text { CR } & \text { Covariation by randomization } \\ \text { UCM } & \text { Uncontrolled manifold } \\ \text { CoM } & \text { Center of mass } \\ X, Y & \text { Fore-aft and lateral dimensions } \\ \text { STEP }(X / Y) & \text { Step parameters (step length/width) } \\ \text { BCOM }(X / Y) & \text { CoM position relative to back foot } \\ & \text { (fore-aft/lateral) } \\ \text { FCOM }(X / Y) & \text { CoM position relative to front foot } \\ & \text { (fore-aft/lateral) } \\ \text { FIX } & \text { Fixed speed condition } \\ \text { OVG } & \text { Overground speed condition } \\ \text { LCS } & \text { Local coordinate system } \\ \text { RCS } & \text { Relative coordinate system } \\ \text { MTPJ } & \text { Metatarsophalangeal joint } \\ \text { TV }, \mathrm{TV} V_{0} & \text { Empirical and decorrelated task variability } \\ \text { COV } & \text { Motor-equivalent covariation } \\ \rho_{X}, \rho_{Y} & \text { Step-CoM correlation (fore-aft/lateral) }\end{array}$

\section{Introduction}

Stability of walking patterns is frequently assessed by means of step-related variability measures, such as step length or step width fluctuations. Increased variability of the step pattern has been found to be associated with increased risk of falling in older adults and with the presence of neurological disorders such as Parkinson's disease (Hausdorff 2005). However, the coordinative mechanisms underlying stabilization of step-related measures and their functional relationship with maintenance of body 
equilibrium remain poorly understood. Gait stability has also been characterized using biomechanical measures, such as whole-body momentum (Kaya et al. 1998; Herr and Popovic 2008; Robert et al. 2009) or the center of mass (CoM) position relative to the center of pressure (Hof 2008; Hof et al. 2005; Lee and Chou 2006). Functionally, CoM position and movement relative to the base of support are directly linked to maintaining equilibrium. Appropriate control of foot placement may serve this function (Gabell and Nayak 1984; Townsend 1985; Redfern and Schumann 1994; Bauby and Kuo 2000), while also, with respect to step length, assuring the spatiotemporal regularity or forward progression (Gabell and Nayak 1984).

Thus, theoretical considerations and empirical findings indicate that step-related as well as CoM-related parameters are controlled during human walking. It has long been observed (Bernstein 1967) that the motor system has more DOF available than are defined by the functional requirements of most motor tasks. This observation has been captured by the terms of motor equivalence or "motor abundance" (Latash 2000). For instance, the length of a step is determined by the configurations of all the major joints of legs and pelvis; an isolated change in any one of these joints induces a change in step length. However, due to motor equivalence, the effect on step length of changes in one joint can be compensated by coordinated changes in other joints. It has been proposed that analyzing the organization of variability across the degrees of freedom (DOF; e.g., joint angles) allows formally testing whether the motor system stabilizes specific task-related variables (TRV) (Scholz and Schöner 1999). The rationale of this approach is that the structure of intrinsic fluctuations in repeated instances of a task is informative about how the motor systems deal with naturally occurring perturbations. Stabilization of a TRV does not necessarily mean that variability is minimized in each of the DOF, but that variability is organized in a way minimizing variability in the TRV. It is important to note that this notion of stabilization is fundamentally different from using raw variability scores of the TRV (such as variance of step parameters) as indicators of stability.

Several methods have been proposed to analyze taskspecific organization of variability in multi-DOF effector systems (Scholz and Schöner 1999; Müller and Sternad 2003; Cusumano and Cesari 2006). The idea common to these approaches is to relate variability at the level of the effector system (e.g., joint angles) to variability of specific TRV. Here, we use a linearized variant of the covariation by randomization (CR) method (Müller and Sternad 2003, 2004). This approach assesses covariation among joint angles by comparing performance (i.e., variability in a specific TRV) between original data and "decorrelated" data, from which all the covariation among joint angles has been removed. In the original $\mathrm{CR}$ method, covariation among joint angles is removed by a randomization procedure. However, for locally linear systems, decorrelation can also be achieved by setting certain entries of the covariance matrix of the elemental variables to zero (Yen and Chang 2009); this is the approach used here. A TRV is considered to be stabilized by motor-equivalent covariation if the empirical variability in that TRV is lower than the (expected) variability in covariation-free data. The larger the increase in empirical TRV variability relative to decorrelated data, the stronger the covariation in the original data set.

Black et al. (2007) analyzed stabilization of CoM and head position relative to the back foot during walking in children using the uncontrolled manifold (UCM) approach (Scholz and Schöner 1999), which represents an alternative way of analyzing the task-specific organization of variability. According to their results, both variables were controlled at times of heel strike, both in the anteriorposterior and mediolateral dimensions. However, UCM effects do not necessarily reflect covariation of the underlying DOF but may also be due to inhomogeneous variability distribution across DOF (Yen and Chang 2009). Comparing the structure of variability in joint angle configurations may be compromised by different TRV depending on different joint angles (e.g., the step length is determined by the joints of the lower body, while the CoM position depends on all major joints of the body). Also, in the Black et al. study, CoM position was only analyzed relative to the back foot, while the position relative to the front foot might be more crucial for body equilibrium at times of heel strike.

The relationship between stabilization of CoM and step position has not yet been analyzed in detail. In particular, the question whether stabilization of foot fall is merely a consequence of stabilization of the CoM relative to each foot or whether there is additional coordination remains to be investigated. Since step length is the arithmetic sum of the anterior-posterior CoM position relative to the back and front feet, respectively, additional stabilization of step length would be indicated by negative step-to-step correlations between the two CoM position measures. The analogous argument can be made for step width. Modeling studies in a passive dynamic walking context indicate that balance during walking is controlled by different mechanisms in the frontal and sagittal plane (Bauby and Kuo 2000; Donelan et al. 2004; O'Connor and Kuo 2009). The present analysis may allow clarifying the relationship between CoM and step control, and potential differences between sagittal and frontal stabilization of body equilibrium.

To address these questions, eight healthy young men (age range 20-30 years) walked on a motorized treadmill 
while their whole-body motion was being recorded. A variety of walking speeds within the usual walking speed range were used to assess generality and potential velocity dependency of observed stabilization patterns. Motorequivalent stabilization of TRV was assessed by the covariation measure at times of heel strike: step length, step width, as well as CoM position relative to the front and back foot in the fore-aft and lateral directions. We predicted that CoM position relative to the front foot would be the TRV with the strongest covariation, because it has the most direct functional link to maintenance of body equilibrium. Moreover, we computed step-to-step correlations between the CoM measures to assess whether CoM positions relative to the back and front feet covaried in a way stabilizing or destabilizing the step parameters.

\section{Methods}

\section{Participants}

Eight healthy young men (mean age: 26.7 years, SD: 2.4 years) took part in the study. Participants were screened by telephone interview for conditions that are known to affect balance or gait performance. Written consent was obtained from the participants before the experiment. Each participant received 10 Euros per hour. The Ethics Committee of the Max Planck Institute for Human Development, Berlin, approved the study.

Prior to the experiment, spontaneous overground walking speed was assessed on a 15-m hallway. Participants were instructed to walk normally, as when walking on a street at a comfortable speed. The measurement was repeated three times for each participant, and the fastest speed was taken as the self-paced overground walking velocity. The mean overground walking speed was $5.27 \mathrm{~km} / \mathrm{h}(\mathrm{SD}=0.29 \mathrm{~km} / \mathrm{h})$.

Apparatus and data acquisition

Kinematic data were measured using a passive infrared reflective marker system (Vicon MX, Oxford, UK; ten cameras, sampling rate $200 \mathrm{~Hz}$ ). Reflective markers were placed on relevant anatomical landmarks according to the VICON Plugin-Gait Fullbody Model (Davis et al. 1991), complemented by one additional marker on each foot (on the fifth metatarsal) to define foot orientation. These included three markers on each foot (two on forefoot and one on heel), four on each leg (lateral epicondyles of knee and ankle, thigh, and shank), four on the pelvis, six on the thorax and shoulder girdle, six on each arm/hand, and four on the head; amounting to a total of 40 markers. The foot markers were placed on prepared sports shoes (provided by the lab); all other markers were applied directly to the skin or attached using straps around wrist and head.

Participants walked on a treadmill (Woodway $\mathrm{GmbH}$, Weil am Rhein, Germany), with the walking area $(200 \times 70 \mathrm{~cm})$ at the level of the surrounding floor. No handrail was present. For safety reasons, a harness was fastened around the waist of the participant and attached to the ceiling. In front of the treadmill, a $200 \times 270 \mathrm{~cm}$ screen was mounted. A virtual environment consisting of a straight path was back-projected on the screen. The visual flow of the virtual environment was synchronized to the speed of the treadmill with an empirically established ratio between visual flow and treadmill speed. Viewed from the center of the treadmill at a height of $1.5 \mathrm{~m}$, the screen approximately covered the visual range of -50 to +50 degrees horizontally and -55 to 25 degrees vertically. During the experiment, the light was switched off in the surrounding room to minimize availability of visual references other than the virtual environment. Moreover, participants were instructed to look straight ahead into the virtual environment.

\section{Design and procedure}

All the participants had prior experience with walking on a treadmill. Still, they were given ample time (at least 10 min of continuous treadmill walking) to get familiar with the treadmill and the virtual environment prior to the experiment. During the familiarization phase, participants walked at a range of walking speeds, including the ones used in the experimental conditions.

Each participant completed a total number of 12 walking trials of 60-s duration each, with two trials for each of six different walking speeds. In the fixed speed (FIX) condition, participants walked at $3.6,4.5$, and $5.4 \mathrm{~km} / \mathrm{h}$. In the overground speed (OVG) condition, participants walked at 80,100 , and $120 \%$ of their spontaneous overground speed, determined prior to the experiment. The FIX and OVG conditions were presented separately, with a resting break of at least $3 \mathrm{~min}$ (or more, if required) in between. In each condition (FIX and OVG), participants walked continuously on the treadmill at different speeds. The speeds were presented either in ascending-descending or descending-ascending order to minimize sequence effects. The order of conditions (FIX and OVG) and speeds (ascending and descending) was counterbalanced between participants.

Prior to each trial, participants were given time to get used to the current speed (according to verbal response from the participant, at least $15 \mathrm{~s}$ ). After this, walking kinematic data were recorded for $60 \mathrm{~s}$. Participants were instructed not to talk, turn their head, or make any additional movements during walking. 
Data analysis and biomechanical model

All the data analyses were performed using custom-written MATLAB (R2007b, The MathWorks Inc.) routines. The kinematic data were bidirectionally lowpass filtered at $10 \mathrm{~Hz}$ with a third-order Butterworth filter. Previous research found that most of the signal in kinematic gait data is below $6 \mathrm{~Hz}$ in typical walking (Winter et al. 1974; Kirtley 2006). We chose a higher frequency to both account for potential differences in cadence across walking speeds and, in particular, preserve kinematic information about the heel strike, which is required to accurately determine step events.

Step events (left/right heel strikes) were determined from vertical acceleration data of foot markers, based on the method described by Pijnappels et al. (2001). Wholebody postures at these time points, denoted by "step postures", were normalized with respect to the position of the back foot (marker at the first metatarsophalangeal joint, MTPJ), so that the position of the MTPJ corresponded to the origin of the global lab coordinate system. Due to missing markers in some trials, step postures could not be determined for all heel strike events. Therefore, we decided to use only the last 20 complete step postures for each foot in each trial. Normalized step postures were submitted to further biomechanical analysis.

A biomechanical model was implemented in MATLAB following the specifications of the Vicon PluginGait model. For the lower body, the PluginGait model is identical to the Conventional Gait Model (CGM, Davis et al. 1991). Local coordinate systems (LCS, represented by an orthogonal matrix and a translation vector) were defined for each relevant body segment of the upper and lower body: foot, shank, thigh, pelvis, chest, upper arms, lower arms, hands, and neck/head. The configuration of each joint is defined by the relative position of the LCS of the two segments meeting at that joint (relative coordinate system, RCS), also represented by a $3 \times 3$ orthogonal matrix and a translation vector. The orthogonal matrix describes the relative orientation of the segments, and the translational vector corresponds to the origin of one LCS relative to the other (these are constant, up to measurement error).

Joint angles were computed by a Cardan decomposition of the corresponding orthogonal matrix (RCS). To avoid fitting non-anatomical angles at the knee joints, the gait model was slightly adjusted (relative to the original PluginGait model) by using the estimated knee joint axis (rather than a shank marker) for defining the LCS of the lower leg. This had the desired effect of removing the torsional component of the knee angles. The remaining non-anatomical abduction/adduction of the knee joint was ignored by replacing the measured abduction/adduction angles by their mean. For the other joints, the computed joint angles were anatomical by definition (i.e., nonanatomical angles were zero in the Cardan decomposition, due to the choice of the LCS and angle sequences). In total, the model comprised 35 joint angles: ankles (2), knees (1), hips (3), lower back (3), neck (3), sternoclavicular joints (2), shoulders (3), elbows (2), and a "joint" at the first MTPJ of the back foot describing the orientation of the foot relative to the floor (3). The floor LCS corresponded to the global lab coordinate system and served as the basis for the model.

For the covariation analysis (see next Section), an accurate forward kinematic model is required, that is, a function $f: E \rightarrow T$ mapping elemental variables (i.e., joint angles; represented in $E$ ) to the respective task-related variables (TRV, e.g., step length; represented in $T$ ). The forward model was defined by reversing the joint angle computation process described above. For a given set of joint angles, RCS was determined by combining the corresponding orthogonal matrices with the (averaged) translational vector. Subsequently, starting from the base segment (the floor at the first MTPJ of the back foot), LCS was computed from the RCS following the kinematic chains across the body. Based on the LCS, the wholebody CoM was determined using the anthropometric measures provided by Winter (2004). Subsequently, the six TRV were computed: Step length (STEPX) and step width (STEPY) were defined as the distance between the toe (MTPJ) marker of the back foot and the heel marker of the front foot, projected to the fore-aft and lateral dimensions, respectively. The positions of the CoM relative to the back (BCOMX and BCOMY) and front foot (FCOMX and FCOMY) were determined relative to these foot markers as well, see Fig. 1. Note that this definition of step length and width differs from the typically used standard measures, which are based on a flat foot position on the ground. Thus, inter-individual differences in foot size and foot placement angle are not taken into account in the mean values of the presently used measures. The relative CoM positions were defined in such a way that they typically have positive values and that STEP $=$ $\mathrm{BCOM}+\mathrm{FCOM}$.

Table 1 summarizes the mean, $\mathrm{SD}$, and model error for each of the TRV. Model errors were similar for the full and the linearized model, justifying the use of the linearized version of the Covariation analysis ${ }^{1}$ (see next Section).

\footnotetext{
${ }^{1}$ For the model including the additional knee adduction/abduction angle, model error was around $2 \mathrm{~mm}$ for all the TRV and thereby substantially smaller for the lateral $(Y)$ measures. To control for the effect of this error, all the analyses reported here were also carried out with a model including knee abduction/adduction, with qualitatively equivalent results.
} 

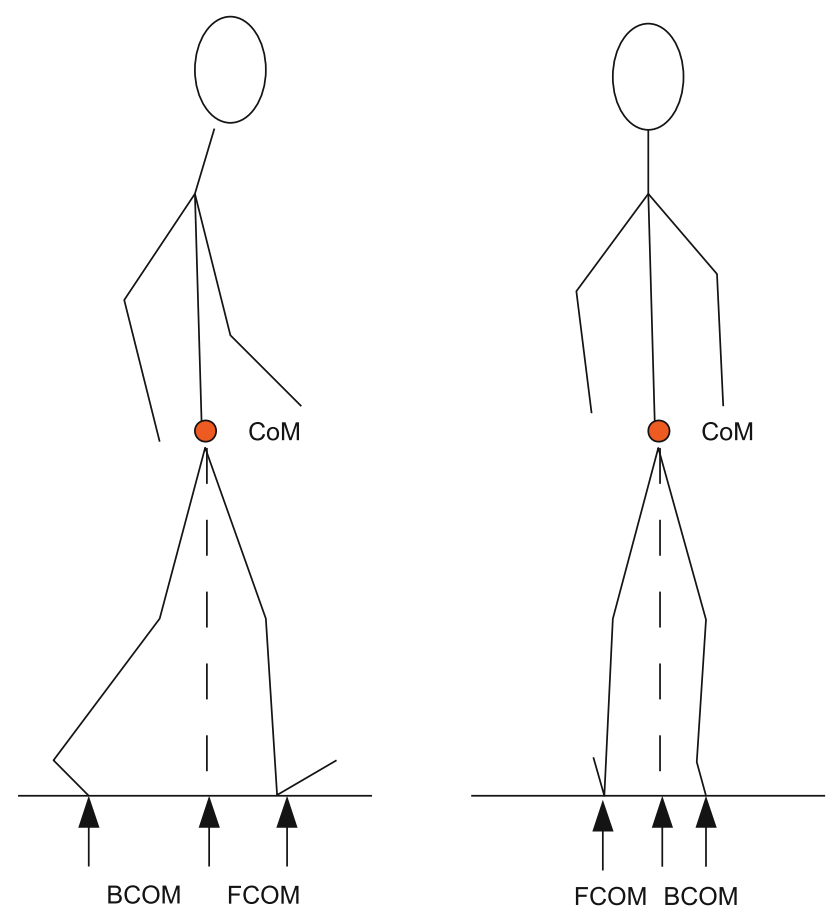

Fig. 1 Illustration of step postures and definition of the TRV: STEPX $=$ FCOMX + BCOMX, STEPY $=$ FCOMY + BCOMY

Covariation analysis

The organization of variability in step postures with respect to the TRV was analyzed with a linearized variant of the CR (Müller and Sternad 2003; Yen and Chang 2009), separately for left and right heel strikes in each trial. In the original CR method, joint angles are decorrelated by randomly and independently permuting the samples of each joint angle. That is, if the joint angle configurations for the 20 steps are represented in a $20 \times 35$ matrix, with samples from each joint angle represented along columns, this procedure consists of rearranging each of the 35 columns by separately applying randomly chosen permutations on 20 elements. The relative increase in variability in the TRV due to decorrelation (determined by means of the forward model) yields a measure of covariation in the original data.
To increase reliability, the randomization procedure needs to be repeated many times (Müller and Sternad 2003).

Here, we use a linearized and computationally more efficient version of this procedure, based on a linearization of the forward model,

$f\left(x-x_{0}\right)=f\left(x_{0}\right)+J\left(x-x_{0}\right)+O\left(\left|x-x_{0}\right|^{2}\right)$

where $J=D f$ is the Jacobian matrix of the forward model $f, x$ denotes any step posture, represented in joint angles, and $x_{0}$ is the averaged step posture in that trial. Since the joint angle space is 35-dimensional and the task space (for each of the TRV) is 1-dimensional, $J$ is a $1 \times 35$-matrix. This approximation also forms the basis for other approaches that quantify the structure of variability in joint angle space (Scholz and Schöner 1999; Cusumano and Cesari 2006; Schöner and Scholz 2007). Based on this approximation, task variability TV (variance in the TRV under consideration) is determined as

$\mathrm{TV}=\operatorname{var}(J x)=J \operatorname{cov}(x) J^{\prime}=J C J^{\prime}$

Note that in this equation, only the covariance matrix of the data set $x, C=\operatorname{cov}(x)$ is used. It can be shown (Yen and Chang 2009, Appendix A) that the expected value of the covariance matrix of decorrelated angles is a matrix $C_{0}$ with the same diagonal entries as $C$, but zeros in all other entries. In the present case, independently permuting all the joint angles is biomechanically inappropriate, since the angles representing, for instance, the configuration of the right hip joint, are not computationally independent. The appropriate randomization procedure in this case would be to apply the same permutation to the angles representing a particular joint (but different permutations to angles from different joints). In this "constrained randomization", covariation is only removed between joint angles from different joints. The corresponding decorrelated covariance matrix $C_{0}$ is a block diagonal matrix, consisting of diagonal quadratic matrices of order 1,2 , or 3 , depending on the number of DOF of the corresponding joint. The elements of $C_{0}$ in these blocks are identical to those of $C$ in the corresponding positions, and all other entries are 0 .

Table 1 Mean and SD of the six TRV, and accuracy of the full and the linearized forward model (RMS between actual and modeled value)

\begin{tabular}{|c|c|c|c|c|c|}
\hline TRV & Dimension & Mean & SD & Deviation & Lin. deviation \\
\hline \multirow[t]{2}{*}{ STEP } & $X$ & $485.45(33.97)$ & $10.60(1.48)$ & $2.88(1.13)$ & $2.89(1.10)$ \\
\hline & $Y$ & $54.93(40.64)$ & 20.79 & $4.20(0.42)$ & $4.26(0.47)$ \\
\hline \multirow[t]{2}{*}{$\mathrm{BCOM}$} & $X$ & $193.00(22.97)$ & 10.10 & $2.32(0.84)$ & $2.33(0.85)$ \\
\hline & $Y$ & $16.22(19.73)$ & $11.35(2.12)$ & $3.18(0.62)$ & $3.32(0.64)$ \\
\hline \multirow[t]{2}{*}{ FCOM } & $X$ & $292.45(24.91)$ & $6.96(0.99)$ & $3.38(1.69)$ & $3.40(1.68)$ \\
\hline & $Y$ & $38.71(22.17)$ & $11.14(2.40)$ & $5.95(0.68)$ & $6.02(0.69)$ \\
\hline
\end{tabular}

The corresponding values were first averaged across all trials and both feet of each participant. The values in the table are between-participant mean and SD (in brackets) 
The decorrelated task variability, $\mathrm{TV}_{0}$, is defined as

$\mathrm{TV}_{0}=J C_{0} J^{\prime}$

Note that both $\mathrm{TV}$ and $\mathrm{TV}_{0}$ are estimated using the linearized forward model. Estimating TV from the original marker data is possible for certain TRV but would increase the influence of modeling and linearization error on the difference between empirical and decorrelated variability.

Determining the Jacobian $J$ using numerical two-point differentiation requires the application of the forward model to $35.2=70$ samples. All subsequent computations are basic linear algebra. In contrast, to get reliable estimates of decorrelated task variability using the CR, the randomization procedure should be repeated many times. The potential increase in model accuracy (for non-linear forward models) comes at the cost of higher computational demands and dependence of the estimate of decorrelated variability on the actually chosen permutations. Note that, in the present situation, the full and linearized forward model hardly differ regarding model accuracy (Table 1), justifying the use of the linearized forward model.

Covariation among joint angles with respect to the TRV under consideration is quantified as the ratio

$\mathrm{COV}=\mathrm{TV}_{0} / \mathrm{TV}$

Covariation is indicated when $\mathrm{TV}_{0}>\mathrm{TV}$, or equivalently, when $\mathrm{COV}>1$. Larger values of $\mathrm{COV}$ reflect stronger covariation. To (empirically) correct for non-normal distribution, statistical analyses are carried out on log-transformed values, and the data will be plotted on a logarithmic scale $^{2}$.

Relationship between CoM and step stabilization

One way to assess the relationship between stabilization of the TRV is to statistically compare the strength of covariation, for instance, covariation with respect to FCOMX versus STEPX. A more direct way of relating stability of CoM and step positions comes from the fact that the step length is the arithmetic sum of the anterior-posterior CoM position relative to the back and front feet, respectively (Fig. 1).

When FCOMX and BCOMX are uncorrelated across the steps within a trial, stability of STEPX results from independent control of (sagittal) CoM positions relative to the feet. In contrast, a negative correlation between FCOMX and BCOMX would indicate that step-to-step fluctuations in one variable are partially compensated by the other in

\footnotetext{
2 In the original $\mathrm{CR}$ approach, a generalized correlation is defined as $\mathrm{TV} / \mathrm{TV}_{0}-1$. To approximate a normal distribution, these measures should be transformed prior to statistical analysis by a variant of Fisher's z'-transformation, in particular when covariation is high. This transformation is essentially equivalent to taking the log transform of COV.
}

their effect on STEPX, indicating additional stabilization of STEPX. On the other hand, a positive correlation between FCOMX and BCOMX would indicate that the influence of fluctuations in one variable on STEPX is amplified by the other, such that STEPX is actually destabilized by the relationship between FCOMX and BCOMX. A similar reasoning applies to the lateral measures STEPY (step width), BCOMY, and FCOMY. Based on these considerations, step-CoM correlations were defined in the fore-aft and lateral directions, separately for each trial and foot (heel strike event), as

$\rho_{X}=\operatorname{corr}(\mathrm{BCOMX}, \mathrm{FCOMX})$

$\rho_{Y}=\operatorname{corr}(\mathrm{BCOMY}, \mathrm{FCOMY})$

By definition, step-CoM correlations are bounded between -1 and 1 .

\section{Statistical analysis}

Statistical analyses were carried out with R (R Development Core Team 2008). The alpha level for statistical significance testing was set to 0.05. Effect sizes for ANOVAs are reported as generalized eta-squared $\eta_{G}^{2}$ (Bakeman 2005).

To correct for non-normal distribution, empirical and decorrelated variability $\left(\mathrm{TV}, \mathrm{TV}_{0}\right)$ as well as covariation for the different TRV were log transformed. The step-CoM correlations were transformed using Fisher's $z$ ' transformation. After transformation, the dependent variables of each participant were averaged across heel strike events (left and right) and trials within the same speed.

Statistical analyses were performed separately for the two walking speed conditions (FIX and OVG). The dependent variables (mean, SD, and covariation for each TRV; step-CoM correlations) were submitted to one-way repeated measures ANOVA separately for FIX and OVG conditions. For each condition, Speed was defined as within-subject factor (three levels; FIX: 3.6, 4.5, and $5.4 \mathrm{~km} / \mathrm{h}$; OVG: OVG - 20\%, OVG, and OVG + 20\%; modeled as linear and quadratic contrasts).

To assess differences in covariation strength between the TRV, the variable COV was submitted to a repeated measures ANOVA with factors Speed (three levels; FIX: 3.6, 4.5, and $5.4 \mathrm{~km} / \mathrm{h}$; OVG: OVG $-20 \%$, OVG, and OVG $+20 \%$; modeled as linear and quadratic contrasts) and TRV (three levels; STEP, BCOM, and FCOM). In addition, covariation for different TRV was compared by means of paired two-tailed $t$ tests, separately at each level of the factor Speed. The alpha level for significance testing in these comparisons was Bonferroni-adjusted to $0.05 / 9$ (number of comparisons within each Condition FIX/OVG and Dimension $X / Y$ ). 
Covariation indices (for the TRV) and step-CoM correlations $(X$ and $Y$ ) were tested against the null hypothesis of having a mean of zero by one-sample $t$ tests at each speed level, with adjusted significance level of $0.05 / 3$ (number of comparisons within each Condition FIX/OVG, Dimension $X / Y$, and TRV).

\section{Results}

The results from the one-way ANOVAs testing the linear and quadratic effects of the factor Speed in different measures (mean, TV, $\mathrm{TV}_{0}, \mathrm{COV}$ ) for the different TRV and in the two conditions (FIX, OVG) are summarized in Table 2. The results are also shown in Fig. 2 (mean), Fig. 3 (empirical and decorrelated variability, plotted as SD rather than variance), and Fig. 4 (covariation).

In the fore-aft direction $(X)$, the means of STEP, BCOM, and FCOM showed linear increases with walking speed. Quadratic effects were smaller or absent. For the lateral $(Y)$ measures, only small effects were found. Empirical fore-aft variability (TV) in all the TRV decreased (linearly) with walking speed in the FIX condition but showed no systematic effects (STEP: linear and quadratic, FCOM: quadratic) in the OVG condition. No significant effects were found for the lateral measures. Decorrelated task variability $\left(\mathrm{TV}_{0}\right)$ for STEPX decreased linearly in FIX and showed a quadratic trend in OVG. For the other TRV, no Speed effects were found in the FIX condition, but increasing linear trends in the OVG condition.

\section{Covariation analysis}

Covariation $(\mathrm{COV})$ in the fore-aft dimension showed linear increasing trends for BCOMX and FCOMX in the FIX condition and for BCOM in the OVG condition, as well as a decreasing linear trend for STEPX in the OVG condition. For the lateral measures, linear increasing trends were found for BCOMY in the FIX condition and for STEPY, BCOMY, and FCOMY in the OVG condition.

Two-tailed $t$ tests comparing the observed covariation distribution against the null hypothesis (log COV has zero

Table 2 Summary of statistical analysis of the DVs for the linear and quadratic effects of speed $[F=F(1,14)]$

\begin{tabular}{|c|c|c|c|c|c|c|}
\hline \multirow[t]{2}{*}{ Measure } & \multirow[t]{2}{*}{ Variable } & \multirow[t]{2}{*}{ Dimension } & \multicolumn{2}{|l|}{ FIX } & \multicolumn{2}{|l|}{ OVG } \\
\hline & & & Speed/linear & Speed/quadratic & Speed/linear & Speed/quadratic \\
\hline MEAN & STEP & $X$ & $F=781.57(0.85)^{*}$ & $F=0.54(0.00)$ & $F=218.41(0.63)^{*}$ & $F=1.80(0.01)$ \\
\hline MEAN & STEP & $Y$ & $F=2.40(0.01)$ & $F=0.45(0.00)$ & $F=0.36(0.00)$ & $F=0.12(0.00)$ \\
\hline MEAN & BCOM & $X$ & $F=913.56(0.92)^{*}$ & $F=1.19(0.01)$ & $F=210.10(0.63)^{*}$ & $F=0.41(0.00)$ \\
\hline MEAN & $\mathrm{BCOM}$ & $Y$ & $F=20.22(0.02)^{*}$ & $F=0.05(0.00)$ & $F=9.21(0.01)^{*}$ & $F=1.09(0.00)$ \\
\hline MEAN & FCOM & $X$ & $F=230.38(0.40)^{*}$ & $F=0.01(0.00)$ & $F=102.62(0.36)^{*}$ & $F=5.03(0.03)^{*}$ \\
\hline MEAN & FCOM & $Y$ & $F=0.24(0.00)$ & $F=0.59(0.01)$ & $F=0.01(0.00)$ & $F=0.02(0.00)$ \\
\hline TV & STEP & $X$ & $F=18.02(0.29)^{*}$ & $F=1.79(0.04)$ & $F=8.47(0.17)^{*}$ & $F=6.60(0.14)^{*}$ \\
\hline $\mathrm{TV}$ & STEP & $Y$ & $F=0.09(0.00)$ & $F=0.46(0.01)$ & $F=1.44(0.01)$ & $F=0.79(0.01)$ \\
\hline TV & $\mathrm{BCOM}$ & $X$ & $F=10.74(0.21)^{*}$ & $F=1.75(0.04)$ & $F=1.84(0.05)$ & $F=3.72(0.09)$ \\
\hline TV & $\mathrm{BCOM}$ & $Y$ & $F=1.36(0.02)$ & $F=1.42(0.02)$ & $F=3.10(0.05)$ & $F=2.52(0.04)$ \\
\hline TV & FCOM & $X$ & $F=20.61(0.24)^{*}$ & $F=3.97(0.06)$ & $F=1.77(0.02)$ & $F=9.51(0.12)^{*}$ \\
\hline TV & FCOM & $Y$ & $F=0.16(0.00)$ & $F=0.78(0.01)$ & $F=0.06(0.00)$ & $F=0.36(0.00)$ \\
\hline TV0 & STEP & $X$ & $F=20.55(0.30)^{*}$ & $F=0.51(0.01)$ & $F=0.66(0.02)$ & $F=9.22(0.18)^{*}$ \\
\hline TV0 & STEP & $Y$ & $F=0.76(0.01)$ & $F=0.45(0.01)$ & $F=23.22(0.23)^{*}$ & $F=0.97(0.01)$ \\
\hline TV0 & BCOM & $X$ & $F=0.02(0.00)$ & $F=1.12(0.01)$ & $F=11.59(0.19)^{*}$ & $F=1.81(0.04)$ \\
\hline TV0 & $\mathrm{BCOM}$ & $Y$ & $F=1.60(0.02)$ & $F=0.38(0.00)$ & $F=24.22(0.13)^{*}$ & $F=6.41(0.04)^{*}$ \\
\hline TV0 & FCOM & $X$ & $F=1.55(0.02)$ & $F=1.03(0.01)$ & $F=7.68(0.13)^{*}$ & $F=3.87(0.07)$ \\
\hline TV0 & FCOM & $Y$ & $F=1.19(0.02)$ & $F=0.17(0.00)$ & $F=34.25(0.22)^{*}$ & $F=2.84(0.02)$ \\
\hline $\mathrm{COV}$ & STEP & $X$ & $F=0.83(0.02)$ & $F=1.00(0.03)$ & $F=12.38(0.32)^{*}$ & $F=0.00(0.00)$ \\
\hline $\mathrm{COV}$ & STEP & $Y$ & $F=1.35(0.02)$ & $F=0.01(0.00)$ & $F=4.74(0.05)^{*}$ & $F=0.08(0.00)$ \\
\hline $\mathrm{COV}$ & BCOM & $X$ & $F=22.80(0.12)^{*}$ & $F=0.29(0.00)$ & $F=16.69(0.11)^{*}$ & $F=0.02(0.00)$ \\
\hline $\mathrm{COV}$ & BCOM & $Y$ & $F=9.85(0.17)^{*}$ & $F=0.47(0.01)$ & $F=13.57(0.14)^{*}$ & $F=0.72(0.01)$ \\
\hline $\mathrm{COV}$ & FCOM & $X$ & $F=16.73(0.12)^{*}$ & $F=1.75(0.01)$ & $F=4.38(0.06)$ & $F=0.13(0.00)$ \\
\hline $\mathrm{COV}$ & FCOM & $Y$ & $F=0.36(0.01)$ & $F=0.15(0.00)$ & $F=15.75(0.17)^{*}$ & $F=0.37(0.00)$ \\
\hline
\end{tabular}

Effect sizes $\left(\eta_{G}^{2}\right)$ are shown in brackets

* Significant effects $(P<0.05)$ 

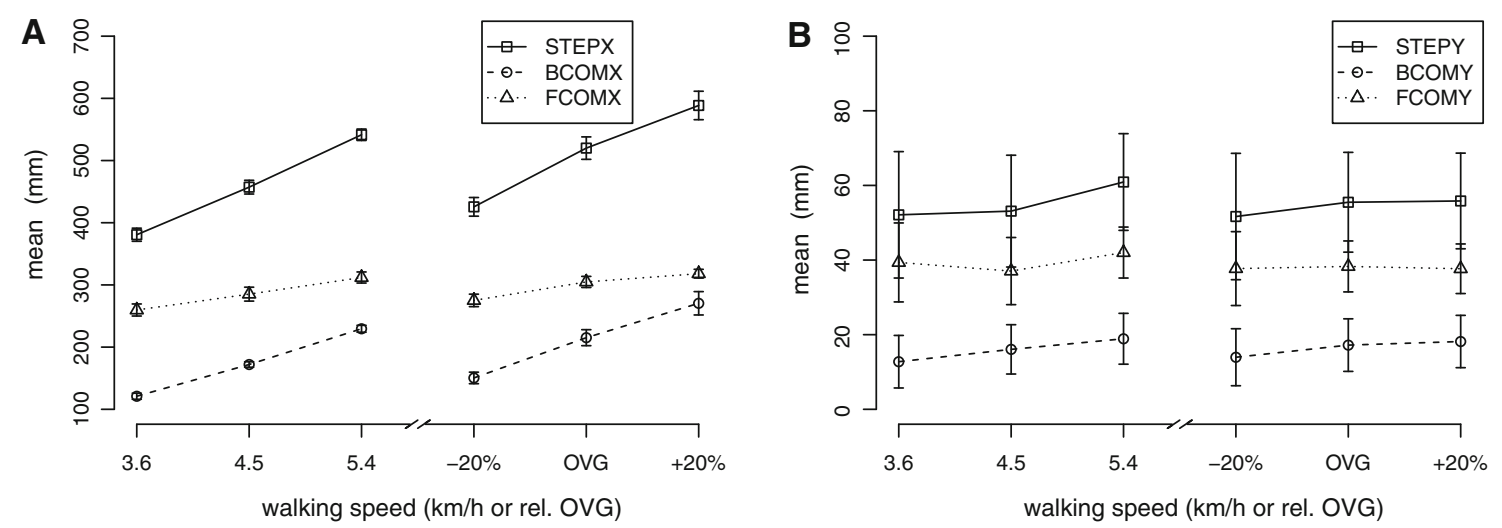

Fig. 2 Mean of the different TRV in fore-aft (a) and lateral (b) directions, as a function of walking speed. Error bars represent SEM
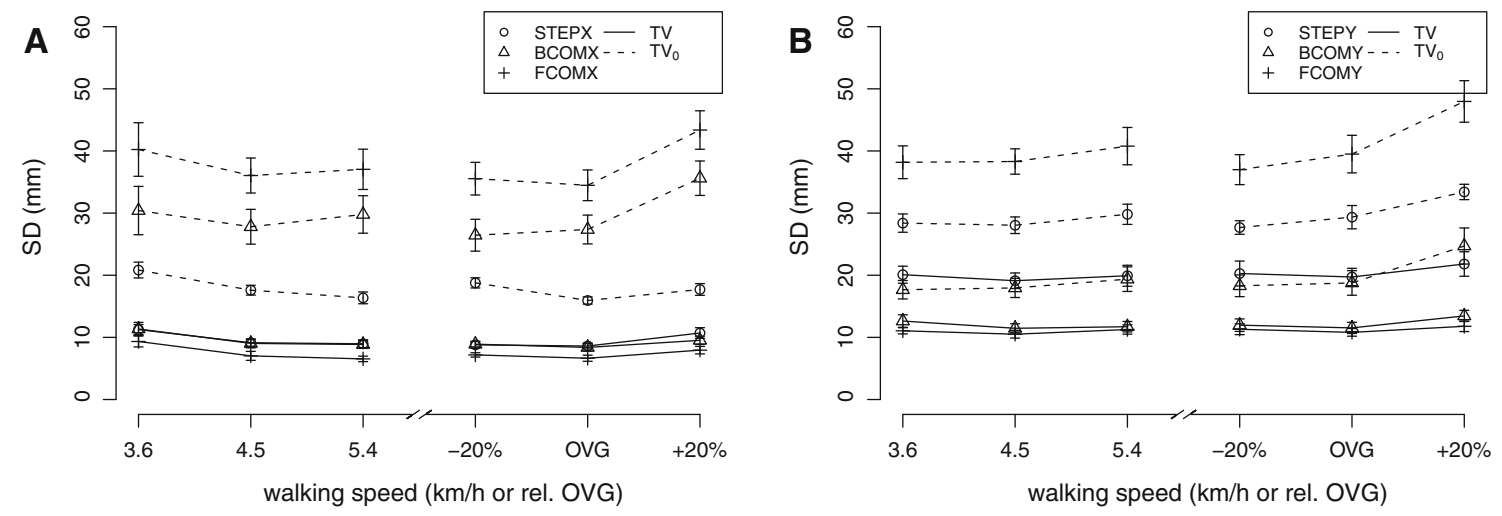

Fig. 3 Empirical and decorrelated variability for the TRV in fore-aft (a) and lateral (b) directions, as a function of walking speed. Variability is represented as standard deviation rather than variance, i.e., the square root of $\mathrm{TV}$ and $\mathrm{TV}_{0}$. Error bars represent SEM
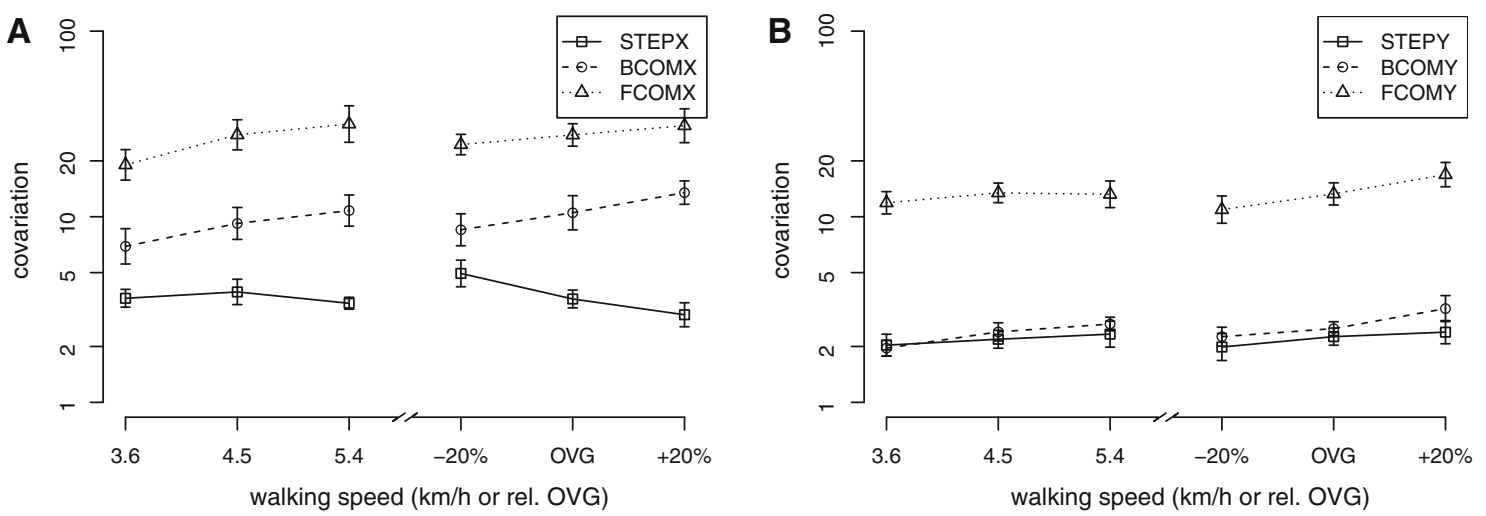

Fig. 4 Covariation index for TRV in fore-aft (a) and lateral (b) directions as a function of walking speed. Error bars represent SEM

mean) were significant for all TRV and for all conditions and walking speeds (Table 5). Thus, covariation was present across all variables, speeds, and conditions.

The results of the ANOVA comparing covariation with respect to different TRV are summarized in Table 3. For both Conditions (FIX and OVG) and both Dimensions ( $X$ and $Y$ ), a significant main effect of TRV was found, with large effect sizes $\left(\eta_{G}^{2}>0.7\right)$. The result of comparing covariation between the TRV by pairwise $t$ tests, separately for each speed, is summarized in Table 4. For the fore-aft dimension $(X)$, covariation was larger for FCOM than for BCOM, and larger for BCOM than for STEP. For the lateral dimension $(Y)$, covariation was stronger for FCOM than for BCOM and STEP but did not differ significantly between STEP and BCOM.

Summing up, the results of the covariation analyses revealed that each of the TRV under consideration was stabilized by motor-equivalent organization of variability 
Table 3 Comparison of covariation between different TRV

\begin{tabular}{lllll}
\hline Cond. & Dimension & TRV & Speed & TRV $\times$ Speed \\
\hline FIX & $X$ & $F(2,14)=133.05(0.769)^{*}$ & $F(2,14)=13.92(0.102)^{*}$ & $F(4,28)=2.62(0.029)$ \\
FIX & $Y$ & $F(2,14)=173.60(0.868)^{*}$ & $F(2,14)=2.31(0.042)$ & $F(4,28)=0.95(0.015)$ \\
OVG & $X$ & $F(2,14)=123.67(0.836)^{*}$ & $F(2,14)=0.65(0.009)$ & $F(4,28)=9.03(0.132)^{*}$ \\
OVG & $Y$ & $F(2,14)=108.37(0.830)^{*}$ & $F(2,14)=10.22(0.123)^{*}$ & $F(4,28)=1.23(0.015)$ \\
\hline
\end{tabular}

* Significant effects $(P<0.05)$

Table 4 Pairwise comparisons of covariation between different TRV at the different speed levels by paired $t$ test $(t=t(7))$

\begin{tabular}{llllllll}
\hline Comparison & Dim & $3.6 \mathrm{~km} / \mathrm{h}$ & $4.5 \mathrm{~km} / \mathrm{h}$ & $5.4 \mathrm{~km} / \mathrm{h}$ & OVG $-20 \%$ & OVG & OVG $+20 \%$ \\
\hline STEP-BCOM & $X$ & $t=-5.25^{*}$ & $t=-5.87^{*}$ & $t=-6.13 *$ & $t=-4.17 *$ & $t=-6.67 *$ & $t=-11.47^{*}$ \\
STEP-FCOM & $X$ & $t=-11.88^{*}$ & $t=-16.70^{*}$ & $t=-10.32^{*}$ & $t=-24.82^{*}$ & $t=-12.85^{*}$ & $t=-10.44^{*}$ \\
BCOM-FCOM & $X$ & $t=-7.66^{*}$ & $t=-11.97^{*}$ & $t=-10.34^{*}$ & $t=-8.62^{*}$ & $t=-5.77 *$ & $t=-6.09 *$ \\
STEP-BCOM & $Y$ & $t=0.48$ & $t=-0.58$ & $t=-0.73$ & $t=-0.85$ & $t=-0.74$ & $t=-1.05$ \\
STEP-FCOM & $Y$ & $t=-12.74^{*}$ & $t=-28.41^{*}$ & $t=-13.23^{*}$ & $t=-12.91^{*}$ & $t=-18.72 *$ & $t=-20.84 *$ \\
BCOM-FCOM & $Y$ & $t=-20.75^{*}$ & $t=-12.47^{*}$ & $t=-11.15^{*}$ & $t=-11.18^{*}$ & $t=-11.97 *$ & $t=-8.96^{*}$ \\
\hline
\end{tabular}

* Significant effects $(P<0.05$, corrected for multiple comparisons $)$

among joint angles. Furthermore, direct comparisons indicate that CoM position relative to the front foot is the most highly prioritized variable, both in the fore-aft and lateral dimensions.

\section{Step-CoM correlations}

Step-CoM correlations in the fore-aft $(X)$ and lateral $(Y)$ directions are shown in Fig. 5a. To give an impression of the reliability of the correlations, the distribution of $P$-values for both measures is plotted in Fig. 5b, pooled over all participants, trials, and feet (heel strike events).

One-way ANOVAs showed a small quadratic effect of speed for STEPY in the FIX condition $[F(1,14)=4.74$, $\left.P<0.05, \eta_{G}^{2}=0.06\right]$ and no reliable effects for any of the other conditions. Table 5 also shows the results of testing step-CoM correlations for deviating from 0 (after

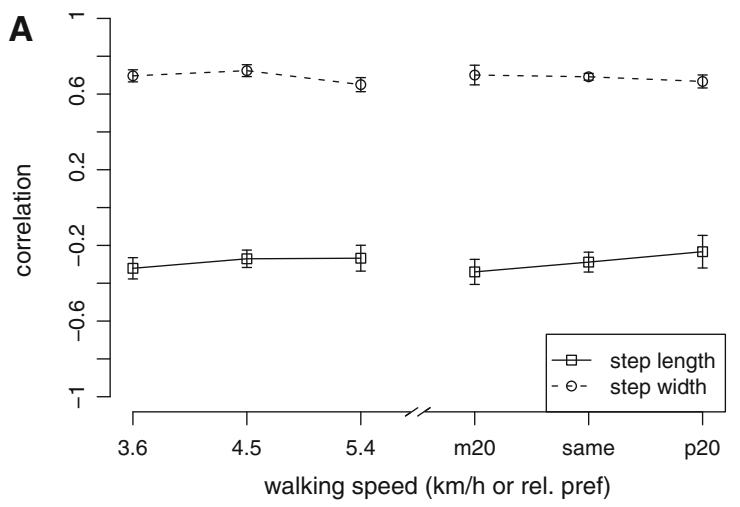

z'-transformation). Opposite effects were found for STEPX and STEPY: Step-CoM correlations with respect to step length tended to be negative, whereas step-CoM correlations with respect to step width were positive across all speeds. This result indicates that the positions of the CoM relative to each foot in the fore-aft direction covaried in a way that stabilized step length. In contrast, a destabilizing step-CoM correlation pattern was found for step width in the lateral direction.

\section{Discussion}

Summary of results

We used a covariation approach to analyze motor-equivalent stabilization of hypothesized task-related variables (TRV)

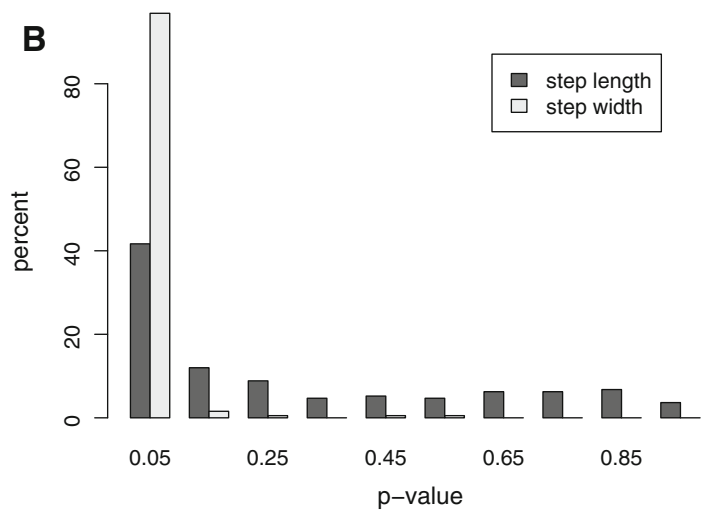

Fig. 5 Step-CoM correlations. a Correlation coefficient, as a function of walking speed and direction (X, Y). Error bars represent SEM. b Histogram of $P$-values for (within-trial, between-step) correlations 
Table 5 Statistical tests (one-sample $t$ tests, $t=t(7)$ ) for the presence of covariation with respect to the different TRV (COV $>1)$, and for the presence of step-CoM correlations $(\rho \neq 0)$

\begin{tabular}{llllllll}
\hline Variable & Dimension & $3.6 \mathrm{~km} / \mathrm{h}$ & $4.5 \mathrm{~km} / \mathrm{h}$ & $5.4 \mathrm{~km} / \mathrm{h}$ & OVG-20\% & OVG & OVG $+20 \%$ \\
\hline STEP & $X$ & $t=11.70^{*}$ & $t=13.09^{*}$ & $t=14.79^{*}$ & $t=16.27^{*}$ & $t=10.83^{*}$ & $t=10.48^{*}$ \\
STEP & $Y$ & $t=5.32^{*}$ & $t=7.24^{*}$ & $t=5.27^{*}$ & $t=3.88^{*}$ & $t=7.20^{*}$ & $t=5.33^{*}$ \\
BCOM & $X$ & $t=8.58^{*}$ & $t=11.21^{*}$ & $t=12.38^{*}$ & $t=10.56^{*}$ & $t=10.95^{*}$ & $t=19.02^{*}$ \\
BCOM & $Y$ & $t=6.87^{*}$ & $t=7.86^{*}$ & $t=11.56^{*}$ & $t=6.77^{*}$ & $t=10.87^{*}$ & $t=7.24^{*}$ \\
FCOM & $X$ & $t=15.54^{*}$ & $t=17.93^{*}$ & $t=15.20^{*}$ & $t=26.69^{*}$ & $t=21.74^{*}$ & $t=16.56^{*}$ \\
FCOM & $Y$ & $t=17.42^{*}$ & $t=21.75^{*}$ & $t=15.51^{*}$ & $t=14.61^{*}$ & $t=18.61^{*}$ & $t=16.36^{*}$ \\
Step-CoM & $X$ & $t=-3.41^{*}$ & $t=-4.45^{*}$ & $t=-3.86^{*}$ & $t=-5.76^{*}$ & $t=-5.07^{*}$ & $t=-2.94$ \\
Step-CoM & $Y$ & $t=21.15^{*}$ & $t=22.17^{*}$ & $t=17.22^{*}$ & $t=13.79^{*}$ & $t=39.22^{*}$ & $t=19.89^{*}$ \\
\hline
\end{tabular}

* Significant effects $(P<0.05$, corrected for multiple comparisons $)$

during walking at the times of heel strike. The covariation analysis, which is here applied for the first time to a complex biomechanical system, served to assess stabilization of TRV by comparing variability in the TRV between empirical and covariation-free (decorrelated) data. The following TRV were analyzed: step length, step width, and center of mass (CoM) position relative to the back foot (BCOM) and front foot (FCOM). Significant covariation was found for each of these TRV across a number of speed conditions and in both the fore-aft and lateral directions. This main finding shows that the TRV under consideration are indeed stabilized by motor-equivalent covariation across the motor system. Moreover, we found evidence for control priorities among these TRV, as the strength of covariation differed between TRV. In both the fore-aft and lateral directions, covariation was highest for FCOM in both. Given that this TRV may be most directly related to maintenance of equilibrium while walking, this result is plausible from a biomechanical point of view. The results regarding the effect of walking speed were mixed. Covariation indices for most TRV showed either no reliable or increasing linear trends with increasing walking speed, indicating that some aspects of walking are more strongly stabilized at higher walking speeds.

An additional analysis assessed, separately for each dimension, whether BCOM and FCOM were coordinated in ways that either stabilized or destabilized foot placement. A clear destabilizing pattern was present in the lateral dimension, where strong positive step-to-step correlations $(\rho Y \approx 0.7)$ were found between BCOMY and FCOMY. It may be argued that stabilization of step width is not a task-relevant goal per se during treadmill walking; instead, the stabilization of step width indicated by the covariation analysis may be a consequence of stabilizing the CoM position relative to each foot in the mediolateral dimension. According to this interpretation, the positive correlation between BCOMY and FCOMY may reflect a shared influence of lateral CoM motion on both measures.
Theoretical considerations and empirical research showing that lateral foot placement is actively controlled to maintain body equilibrium support this interpretation (Gabell and Nayak 1984; Bauby and Kuo 2000; Hof et al. 2005). This line of reasoning validates the use of step width variability as an indicator of postural instability during walking (Gabell and Nayak 1984; Owings and Grabiner 2004), as increased variability in lateral foot placement is likely to reflect corrective responses to balance perturbations.

In contrast, in the fore-aft dimension, BCOMX and FCOMX showed, on average, negative inter-step correlations, indicating that STEPX was stabilized in addition to CoM position. This suggests that stabilization of step length may be an additional task-relevant goal during walking, assuring, for instance, spatiotemporal regularity of the gait pattern (Gabell and Nayak 1984). However, as mentioned above, a direct comparison of the corresponding covariation strengths showed that $\mathrm{CoM}$ stabilization was prioritized over this goal.

\section{Theoretical and methodological considerations}

The present analysis goes beyond previous work on gait variability in several respects. Variability of gait patterns is frequently assessed using step-related measures, such as the standard deviation of step length or step width. Empirically, these measures have been found to be associated with gait stability and to show changes with healthy aging as well as well as functional decline due to neurological disease (Hausdorff 2005; Hausdorff et al. 1997, 2001; Owings and Grabiner 2004). However, it was not known whether these variables are actually stabilized by the motor system in a formal sense. Moreover, previous research has not directly examined the relationship between these step-related measures and functionally more critical measures, such as the CoM position relative to the base of support. 
We operationalized "stabilization" in two ways: first, covariation among the major joints of the body was analyzed with respect to each of the six CoM- and steprelated variables (Müller and Sternad 2003), yielding a quantitative measure of coordination among these joints with respect to stabilization of the TRV. Importantly, stabilization of a TRV was not assessed based on the amount of variability per se, but by comparing the variance in the TRV to the variance predicted when covariation among joints is eliminated by the decorrelation procedure. Thus, stabilization in this sense means that step-to-step fluctuations in individual joints are not independent but are organized in a way that minimizes variance in the TRV. Second, step-CoM correlations were used to examine how strongly the relative positions of the CoM to the back and front feet were coordinated, and, if so, in a way that would either stabilize or destabilize the step parameters (step length and width). Our results confirm that step parameters are stabilized in a formal sense by inter-joint covariation, albeit to a lesser extent than the CoM relative to the front foot. Negative step-CoM correlations in the fore-aft direction indicate that step length is stabilized beyond CoM stabilization, while positive correlations in the lateral direction suggest that step width is controlled mainly to stabilize body equilibrium in the frontal plane.

The number of steps used to assess variability and stabilization indices for each speed condition presents a limitation of the present study. It has been suggested that step-related variability measures reach a stationary level only after walking at least 400 steps during treadmill walking (Owings and Grabiner 2003). The actual computation of variability scores in that study was based on the last $10 \%$ of the step series (about 70 steps). However, we do not think that our results are compromised by the fact that we used a lower amount of steps for the following reasons. First, the main question addressed by Owings and Grabiner (2003) concerns how many steps a subject should walk on a treadmill before meaningful step variability measures can be obtained and not how many steps are required to actually compute a variability index. Second, we performed the same analysis with all available steps included (for most subjects, full marker data were available for 50-70 steps per foot and trial). The results of this analysis were qualitatively the same as those reported here. Third, the results were consistent across a range of walking speeds. Finally, the main goal of our study was not to determine step pattern variability but to assess the motor-equivalent organization of variability across the body. Previous studies addressing this kind of question, for instance with respect to manual pointing movements (Tseng et al. 2002, 2003), have used a similar number (typically around 20) of movement repetitions as used by us.
Control priorities between different TRV have previously been analyzed using the UCM approach (Latash et al. 2007) that contrasts goal-equivalent and non-goal-equivalent components of joint angle variability. For instance, during sit-to-stand movements, the CoM trajectory was found to be more strongly stabilized than the head or hand trajectory (Scholz and Schöner 1999). The UCM approach is, in some respects, similar to the CR method used here. However, the two approaches differ regarding the question to what extent variability in DOF contributing little or not at all to the TRV is taken into account. In the CR approach, such DOF are largely ignored (since variability is assessed in task space), but they may affect the UCM index (Schöner and Scholz 2007). Since different DOF contributed to very different extents to the TRV under consideration here, the CR method seemed to be methodologically preferable for the purpose of the present study. (We note, parenthetically, that the analyses reported here were also carried out using the UCM approach that yielded qualitatively equivalent results).

Hof et al. (2005) proposed a biomechanical criterion for stability of walking based on the extrapolated CoM, which is a measure combining CoM position and velocity (see also Townsend 1985). CoM velocity in the fore-aft dimension is relatively constant during treadmill walking (and dominated by the velocity of the treadmill). In contrast, variability in the lateral dimension is likely to be higher and to have a shared effect on BCOMY (past movement relative to the back foot) and FCOMY (active control of lateral foot placement), which may explain the positive correlation observed between these measures. The necessity of active control of lateral foot placement is also consistent with a simulation study in a passive dynamic walking framework (Bauby and Kuo 2000). It remains to be investigated to what extent stabilization of step length beyond CoM stabilization observed here reflects active neuromuscular control and to what extent it may be due to passive or low-level (e.g., propriospinal) mechanisms (Bauby and Kuo 2000). To our knowledge, motor-equivalent stabilization (in the sense of covariation used here) has not yet been investigated with passive dynamic walking models, and such studies may shed light on the issue of passive and active contributions in the stabilization of step length.

Besides the above mentioned approaches based on biomechanical and variability measures, stability of walking patterns has also been assessed in perturbation studies as well as by using "dynamic stability" measures analyzing the temporal structure of variability. Applying external perturbations during walking, such as an unpredictable movement of the support surface, allows assessing the efficiency of balance-preserving responses, thereby providing a direct measure of stability (e.g., Tang and Woollacott 1998). 
On the other hand, dynamic measures assess how small, naturally occurring perturbation evolves in time. Dynamic stability is indicated when deviations from a mean pattern or differences between nearby trajectories tend to be reduced over time (Dingwell and Cusumano 2000; Dingwell et al. 2001; England and Granata 2007; Kang and Dingwell 2008). This dynamic definition of stability is different from the notion of motor-equivalent stabilization employed here, but both are based on the analysis of the structure of variability induced by intrinsic perturbations ("noise") of the motor system: dynamic measures analyze how deviations are attenuated over time, while covariation assesses motorequivalent compensation for deviations in different DOF of the motor system. The relationship of these two notions of stability to each other has not yet been investigated to our knowledge. An interesting difference between the two kinds of measures is seen in the effect of walking speed: while covariation indices for most TVR showed no reliable trend or increased with walking speed in the present study, dynamic stability measures have been found to decrease with walking speed (Dingwell and Marin 2006; Kang and Dingwell 2008). Moreover, the relations of both kinds of stability indices to stability in the sense of effectively responding to external perturbations are presently unknown and present important targets for future research.

The mechanics of walking has been described by an inverted pendulum approximation (Cavagna et al. 1977), which is still used in current models of locomotion (Kuo 2007; Griffin et al. 2004). Full and Koditschek (1999) refer to the inverted pendulum model as a "template" providing an approximative description of the body's behavior, which however should be complemented by investigating the underlying coordinative mechanisms. Chang et al. (2009) recently found that the individual joint angles in the cat hind limb are coordinated in a way that stabilizes wholelimb length and orientation. Moreover, peripheral nerve injury to the ankle extensor muscle resulted in marked acute and chronic changes in joint angle patterns during the step cycle but left whole-limb kinematics (leg length and orientation) largely intact. This suggests that intra-limb coordination stabilizes whole-limb kinematics (templates), which may be the basis for efficient and robust hierarchical control of locomotion (Chang et al. 2009) under unperturbed as well as perturbed conditions. This view suggests an alternative (but not exclusive) interpretation of our present results according to which stabilization of the CoM relative to the back and front feet may be a consequence of a whole-limb control mechanism of the legs during locomotion.

Our study also contributes in the methodological domain. We assessed motor-equivalent stabilization of TRV by a linearized variant of the covariation by randomization (CR) method (Müller and Sternad 2003). This demonstrates the applicability of this method in the context of biomechanically complex movements; to our knowledge, the CR method has previously been applied mainly to lowdimensional models, such as a (two-dimensional) virtual skittles task (Müller and Sternad 2004). The presently used linearization reduces the computational costs of applying the CR technique, which is particularly relevant for high-dimensional models. We also propose a way of adapting the CR and (its linearized version) in case of biomechanically inter-dependent joint angles, such as the three angles representing the configuration of the hip joint. In this situation, which may be problematic for the CR method in its original formulation (Schöner and Scholz 2007), the corresponding joint angles should be decorrelated conjointly (i.e., relative to the other angles, but not relative to one another).

With respect to external validity, we note that the present results were obtained with a treadmill paradigm and may not fully generalize to overground walking. We took several measures to decrease the differences between treadmill and overground walking. First, the range of walking speeds included speeds that corresponded to participants' individual overground walking speed. Second, the treadmill was embedded into the ground of the building, so that individuals walked at the level of the surrounding floor. Third, a virtual environment consisting of a straight path was projected on a screen to provide a more naturalistic context for walking. Using a similar experimental setup, Schellenbach et al. (2010) recently reported that treadmill walking after familiarization closely resembled walking patterns observed in overground walking. Based on these considerations, we are confident that the main findings of the present study generalize to overground walking.

\section{Conclusion}

To summarize, we studied the relationship between the control of step parameters and CoM position relative to the base of support. All the task-related variables under consideration were stabilized by motor-equivalent covariation of joint angles across the body. Whether this stabilization was due to active control of the central nervous system, and to what extent it may also due to passive dynamic mechanisms, cannot be answered from the present analysis. Covariation was strongest with respect to CoM position relative to the front foot, suggesting that this variable has the highest control priority. Additional correlation analyses between these variables indicate that step length may be a controlled variable per se, for instance to assure spatiotemporal regularity of the gait pattern, while lateral foot placement seems to mainly serve maintenance of body equilibrium. 
Acknowledgments We would like to thank Michael Schellenbach, Gabriele Faust, and Sabine Schaefer, as well as the student assistants of the Sensorimotor-Cognitive Couplings Team at the MPI for Human Development for organizational and technical support and help with data acquisition and processing. JV is supported by a scholarship from the International Max Planck Research School LIFE (The Life Course: Evolutionary and Ontogenetic Dynamics).

\section{References}

Bakeman R (2005) Recommended effect size statistics for repeated measures designs. Behav Res Methods 37(3):379-384

Bauby CE, Kuo AD (2000) Active control of lateral balance in human walking. J Biomech 33(11):1433-1440

Bernstein N (1967) The coordination and regulation of movements. Pergamon, Oxford

Black DP, Smith BA, Wu J, Ulrich BD (2007) Uncontrolled manifold analysis of segmental angle variability during walking: preadolescents with and without down syndrome. Exp Brain Res 183(4):511-521

Cavagna GA, Heglund NC, Taylor CR (1977) Mechanical work in terrestrial locomotion: two basic mechanisms for minimizing energy expenditure. Am J Physiol 233(5):R243-R261

Chang Y-H, Auyang AG, Scholz JP, Nichols TR (2009) Whole limb kinematics are preferentially conserved over individual joint kinematics after peripheral nerve injury. J Exp Biol 212(Pt 21): 3511-3521

Cusumano J, Cesari P (2006) Body-goal variability mapping in an aiming task. Biol Cybern 94:367-379

Davis RB, Ounpuu S, Tyburski D, Gage JR (1991) A gait analysis data collection and reduction technique. Hum Mov Sci 10:5

Dingwell JB, Cusumano JP (2000) Nonlinear time series analysis of normal and pathological human walking. Chaos 10(4):848-863

Dingwell JB, Marin LC (2006) Kinematic variability and local dynamic stability of upper body motions when walking at different speeds. J Biomech 39(3):444-452

Dingwell JB, Cusumano JP, Cavanagh PR, Sternad D (2001) Local dynamic stability versus kinematic variability of continuous overground and treadmill walking. J Biomech Eng 123(1):27-32

Donelan JMJM, Shipman DWDW, Kram R, Kuo ADAD (2004) Mechanical and metabolic requirements for active lateral stabilization in human walking. J Biomech 37(6):827-835

England SA, Granata KP (2007) The influence of gait speed on local dynamic stability of walking. Gait Posture 25(2):172-178

Full RJ, Koditschek DE (1999) Templates and anchors: neuromechanical hypotheses of legged locomotion on land. J Exp Biol 202(Pt 23):3325-3332

Gabell A, Nayak US (1984) The effect of age on variability in gait. J Gerontol 39(6):662-666

Griffin TM, Main RP, Farley CT (2004) Biomechanics of quadrupedal walking: how do four-legged animals achieve inverted pendulum-like movements? J Exp Biol 207(Pt 20):3545-3558

Hausdorff JM (2005) Gait variability: methods, modeling and meaning. J Neuroeng Rehabil 2:19

Hausdorff JM, Edelberg HK, Mitchell SL, Goldberger AL, Wei JY (1997) Increased gait unsteadiness in community-dwelling elderly fallers. Arch Phys Med Rehabil 78(3):278-283

Hausdorff JM, Rios DA, Edelberg HK (2001) Gait variability and fall risk in community-living older adults: a 1-year prospective study. Arch Phys Med Rehabil 82(8):1050-1056

Herr H, Popovic M (2008) Angular momentum in human walking. J Exp Biol 211(Pt 4):467-481

Hof AL (2008) The 'extrapolated center of mass' concept suggests a simple control of balance in walking. Hum Mov Sci 27(1):112-125
Hof AL, Gazendam MGJ, Sinke WE (2005) The condition for dynamic stability. J Biomech 38(1):1-8

Kang HG, Dingwell JB (2008) Effects of walking speed, strength and range of motion on gait stability in healthy older adults. J Biomech 41(14):2899-2905

Kaya BK, Krebs DE, Riley PO (1998) Dynamic stability in elders: momentum control in locomotor ADL. J Gerontol A Biol Sci Med Sci 53(2):M126-M134

Kirtley C (2006) Clinical gait analysis: theory and practice. Churchill Livingstone, New York

Kuo AD (2007) The six determinants of gait and the inverted pendulum analogy: a dynamic walking perspective. Hum Mov Sci 26(4):617-656

Latash M (2000) There is no motor redundancy in human movements. There is motor abundance. Mot Control 4(3):259-260

Latash ML, Scholz JP, Schöner G (2007) Toward a new theory of motor synergies. Mot Control 11:276-308

Lee H-J, Chou L-S (2006) Detection of gait instability using the center of mass and center of pressure inclination angles. Arch Phys Med Rehabil 87(4):569-575

Müller H, Sternad D (2003) A randomization method for the calculation of covariation in multiple nonlinear relations: illustrated with the example of goal-directed movements. Biol Cybern 89(1):22-33

Müller H, Sternad D (2004) Decomposition of variability in the execution of goal-oriented tasks: three components of skill improvement. J Exp Psychol Hum Percept Perform 30(1): 212-233

O'Connor SM, Kuo AD (2009) Direction-dependent control of balance during walking and standing. J Neurophysiol 102(3): $1411-1419$

Owings TM, Grabiner MD (2003) Measuring step kinematic variability on an instrumented treadmill: how many steps are enough? J Biomech 36(8):1215-1218

Owings TM, Grabiner MD (2004) Step width variability, but not step length variability or step time variability, discriminates gait of healthy young and older adults during treadmill locomotion. J Biomech 37(6):935-938

Pijnappels M, Bobbert MF, van Dieën JH (2001) Changes in walking pattern caused by the possibility of a tripping reaction. Gait Posture 14(1):11-18

Redfern MS, Schumann T (1994) A model of foot placement during gait. J Biomech 27(11):1339-1346

Robert T, Bennett BC, Russell SD, Zirker CA, Abel MF (2009) Angular momentum synergies during walking. Exp Brain Res 197(2):185-197

Schellenbach M, Lövdén M, Verrel J, Krüger A, Lindenberger U (2010) Adult age differences in familiarization to treadmill walking within virtual environments. Gait Posture 31(3):295-299

Scholz JP, Schöner G (1999) The uncontrolled manifold concept: identifying control variables for a functional task. Exp Brain Res 126(3):289-306

Schöner G, Scholz JP (2007) Analyzing variance in multi-degree-offreedom movements: uncovering structure versus extracting correlations. Mot Control 11(3):259-275

Tang PF, Woollacott MH (1998) Inefficient postural responses to unexpected slips during walking in older adults. J Gerontol A Biol Sci Med Sci 53(6):M471-M480

R Development Core Team (2008). R: a language and environment for statistical computing. R Foundation for Statistical Computing, Vienna, Austria. ISBN 3-900051-07-0

Townsend MA (1985) Biped gait stabilization via foot placement. J Biomech 18(1):21-38

Tseng Y, Scholz JP, Schoner G (2002) Goal-equivalent joint coordination in pointing: affect of vision and arm dominance. Mot Control 6(2):183-207 
Tseng YW, Scholz JP, Schoner G, Hotchkiss L (2003) Effect of accuracy constraint on joint coordination during pointing movements. Exp Brain Res 149(3):276-288

Winter DA (2004) Biomechanics and control of human movement. Human Kinetics, Champaign
Winter DA, Sidwall HG, Hobson DA (1974) Measurement and reduction of noise in kinematics of locomotion. J Biomech 7(2):157-159

Yen JT, Chang Y-H (2009) Rate-dependent control strategies stabilize limb forces during human locomotion. J R Soc Interface 7(46):801-810 